\title{
Heberling, Martin (2017): Perspektiven der räumlichen Steuerung des Einzelhandels: Das Dilemma kommunaler Selbstverwaltung und die Grenzen der Raumordnung
}

\author{
Essen: Klartext Verlag. $=$ Dortmunder Beiträge zur Raumplanung 146. $238 \mathrm{~S}$.
}

\section{Frank Osterhage ${ }^{1} \mathbb{D}$}

Online publiziert: 12. Februar 2018

๑) Springer-Verlag GmbH Deutschland, ein Teil von Springer Nature 2018

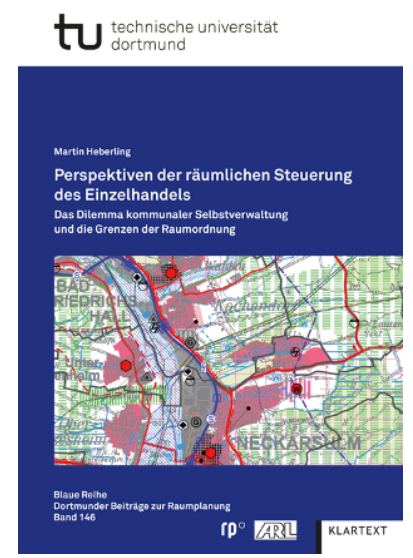

Kaum ein anderes Thema der räumlichen Planung beschäftigt die Praxis so intensiv wie die Steuerung des Einzelhandels. Mittlerweile finden seit mehreren Jahrzehnten umfangreiche Bemühungen auf den Ebenen der kommunalen Bauleitplanung sowie der Regional- und Landesplanung statt, um Einzelhandelsvorhaben auf geeignete Standorte zu lenken. Die Anstrengungen sind mit der Zielsetzung verbunden, die Erreichbarkeit von Versorgungsangeboten für alle Bevölkerungsgruppen zu gewährleisten und auf verkehrsminimierende Siedlungsstrukturen hinzuwirken. Es ist jedoch offensichtlich, dass die tatsächliche Raumentwicklung nicht selten von diesen weithin akzeptierten Zielen abweicht.

Diese Beobachtung bildet den Ausgangspunkt für das Buch von Martin Heberling, das im Rahmen seiner Dissertation an der Fakultät Raumplanung an der Technischen

Frank Osterhage

frank.osterhage@ils-forschung.de

1 ILS - Institut für Landes- und Stadtentwicklungsforschung, Brüderweg 22-24, 44135 Dortmund, Deutschland
Universität Dortmund entstanden ist. Mit der Veröffentlichung stößt er in eine Lücke. Denn trotz der großen Bedeutung des Themas in der Planungspraxis gibt es erstaunlich wenige wissenschaftliche Arbeiten, in denen die zur Anwendung kommenden Regelungen auf den Prüfstand gestellt und tiefer gehende Überlegungen $\mathrm{zu}$ den inhaltlichen Grundlagen der Einzelhandelssteuerung vorgenommen werden. Mit dieser Intention knüpft das Buch an Beiträge an, die sich bereits vor einigen Jahren um eine konzeptionelle Erneuerung der maßgeblichen Steuerungskomponenten bemüht haben. Hierbei ist vor allem an den umfassenden Bericht eines ARL-Arbeitskreises zur „Fortentwicklung des Zentrale-Orte-Konzepts“ (Blotevogel 2002) zu erinnern.

Heberling nimmt bei seiner Arbeit $\mathrm{zu}$ Beginn eine Auseinandersetzung mit dem Steuerungsgegenstand „Einzelhandel“" vor, um eine definitorische Grundlage für die weitere Untersuchung zu schaffen (Kapitel 2). Es erfolgt eine Abgrenzung zum Großhandel und eine Unterteilung nach Sortimentsgruppen. Den Kern dieses Kapitels bildet jedoch eine innere Differenzierung der Nutzungsart Einzelhandel. Unter Berücksichtigung der Baunutzungsverordnungen 1962 bis 2013 werden alle im Planungsrecht genannten Betriebstypen dargestellt und drei Kategorien zugeordnet, die sich hinsichtlich der Frage der Zulassungsfähigkeit unterscheiden.

Anknüpfend an diese Typenbildung werden detailliert die Möglichkeiten von Kommunen erörtert, die Standorte für Einzelhandelsbetriebe im Rahmen ihrer Bauleitplanung räumlich zu steuern (Kapitel 3). Das Ergebnis dieser systematischen Betrachtung fällt eindeutig aus: „Ein Defizit an Steuerungsmöglichkeiten ist nicht auszumachen“ (S. 111). Gemeinden können demnach exakt definieren, wo sich in ihrem Gemeindegebiet Einzelhandelsvorhaben realisieren lassen - und wo dies nicht möglich ist. Es wird plausibel 
dargelegt, dass sie bei sachgerechter Nutzung der vorhandenen Steuerungskomponenten und Steuerungsinstrumente in der Lage sind, parzellenscharfe Bestimmungen über die zulässige Verkaufsfläche und das zulässige Sortiment festzusetzen.

Dennoch kommt es immer wieder dazu, dass Einzelhandelsstandorte entwickelt werden, die den eigentlich bestehenden städtebaulichen Zielen einer Gemeinde nicht entsprechen. Die Ursache hierfür ist nach Überzeugung des Autors auf ein kommunales Dilemma zurückzuführen, das aus der Konkurrenzsituation zwischen Gemeinden bei Ansiedlungsvorhaben resultiert. Mithilfe einer spieltheoretischen Analyse wird deutlich gemacht, dass eine Kommune - aufgrund der Unsicherheit im Hinblick auf das Verhalten der Nachbargemeinde - rational handelt, wenn sie ihre eigenen planerischen Zielvorstellungen zurückstellt und den Interessen eines Investors entgegenkommt. Im Ergebnis werden lediglich zweitbeste Lösungen umgesetzt, und die von der Gemeinde selbst ursprünglich favorisierten Standorte kommen nicht zum Zuge. Heberling spricht diesbezüglich auch von einer „Dysfunktionalität der kommunalen Selbstverwaltung“ (S. 188).

Hieraus wird die Notwendigkeit einer überkommunalen Steuerung abgeleitet, die die im Wettbewerb stehenden Gemeinden gewissermaßen voreinander schützt. Um die existierende Zwangslage zu überwinden, bedarf es offensichtlich einer Regelung, die der kommunalen Planungsentscheidung vorgelagert und für alle Gemeinden verbindlich ist. Erst dadurch werden Kommunen nach der aufgezeigten Logik in die Lage versetzt, ihre eigene Plankonzeption gegenüber Investoreninteressen durchzusetzen.

Für diese Aufgabe scheint im Grunde die Raumordnung als überörtliche und überfachliche Planungsebene prädestiniert, weshalb im folgenden Abschnitt ihre Möglichkeiten und Grenzen bei der Einzelhandelssteuerung behandelt werden (Kapitel 4). Gegenstand der Untersuchung sind das Zentrale-Orte-Konzept als normatives Plankonzept sowie die damit verknüpften Steuerungskomponenten Konzentrationsgebot, Integrationsgebot, Beeinträchtigungsverbot und Kongruenzgebot. Als Resultat einer kritischen Überprüfung kommt der Autor zu der Schlussfolgerung, dass eine wirksame Steuerung von raumbedeutsamen Einzelhandelsnutzungen durch die Raumordnung möglich ist, sofern Raumstrukturen mit einer klaren hierarchischen Ordnung vorliegen. Für verdichtete Räume stellt er hingegen fest, dass die vorherrschenden Steuerungskomponenten an Grenzen stoßen und dort nicht angewendet werden können.

Insbesondere die zentralörtliche Funktionszuweisung (für das ganze Gemeindegebiet) und die Definition eines Verflechtungsbereichs sind nach der vorgebrachten Erläuterung in Verdichtungsräumen nur schwer zu argumentieren. Eine nachvollziehbare Festlegung und Priorisierung von geeigneten Einzelhandelsstandorten mit den Mitteln eines
Zentrale-Orte-Konzepts ist in einer solchen Situation laut Heberling kaum machbar, da die Siedlungsstrukturen häufig über Gemeindegrenzen hinweg zusammengewachsen sind und nahezu jeder Raumpunkt über das öffentliche Verkehrsnetz erreichbar ist: „Die raumordnerischen Ziele zu erreichen, ist in verdichteten Räumen grundsätzlich an vielen Standorten möglich“ (S. 171). Diese Problematik wird vom Autor als Dilemma der Raumordnung bezeichnet.

Als nächster Untersuchungsschritt folgt die Suche nach einer Lösung für die bei der Steuerung des Einzelhandels ausgemachten Dilemmata der Raumplanung (Kapitel 5). Zunächst werden verschiedene Praxisbeispiele für Steuerungsansätze auf der über- und zwischengemeindlichen Ebene unter die Lupe genommen: die Integration eines Standortkonzepts in den Regionalplan der Region Stuttgart, der Regionale Flächennutzungsplan des Ballungsraums Frankfurt/Rhein-Main, der Gemeinsame Flächennutzungsplan des Raums Kassel und das Regionale Einzelhandelskonzept des östlichen Ruhrgebiets. Wie zu erwarten, lässt sich keine pauschale Empfehlung für einen dieser Ansätze aussprechen. Nach einer Auseinandersetzung mit den Vor- und Nachteilen der einzelnen Modelle regt Heberling jedoch als favorisierte Möglichkeit an, mit einem sachlichen Teilflächennutzungsplan auf der Grundlage einer interkommunalen Vereinbarung zu arbeiten.

Da über- und zwischengemeindliche Handlungsansätze noch immer eher eine Ausnahme darstellen, wird anschlieBend diskutiert, welche Rolle der Raumordnung trotz des bestehenden Dilemmas bei der Steuerung des Einzelhandels in verdichteten Räumen zukommen kann. Im Ergebnis hält der Autor es für sinnvoll, vor allem das raumordnerische Beeinträchtigungsverbot beizubehalten. Zudem spricht er sich für ein mit den Gemeinden abgestimmtes Integrationsgebot aus, das angesichts der besonderen Verhältnisse in Verdichtungsräumen auch Ausnahmen zulässt. Nach seiner Einschätzung sollte dagegen auf das Konzentrationsgebot und das Kongruenzgebot in verdichteten Räumen verzichtet werden, da für sie dort eine überzeugende inhaltliche Rechtfertigung fehlt.

Mit der vorliegenden Publikation leistet Heberling einen wertvollen Beitrag zum Themenfeld der Handels- und Planungsforschung. Zum einen wird herausgearbeitet, worin die gegenwärtigen Defizite bei der räumlichen Steuerung des Einzelhandels bestehen. Die Arbeit geht zum anderen deutlich über diese Defizitanalyse hinaus, da sie auf die Entwicklung von konstruktiven Lösungsansätzen ausgerichtet ist. Hierbei scheut sich der Autor nicht davor, bei strittigen Fragen eine klare eigene Position zu beziehen. Im Ergebnis liefert das Buch viele konstruktive Anregungen für eine Diskussion um die Fortentwicklung des derzeitigen Steuerungssystems. Es ist daher zu hoffen, dass es eine hohe Aufmerksamkeit bei allen Akteuren erfährt, die sich auf kommunaler und überkommunaler Ebene mit 
großem Engagement der Einzelhandelssteuerung durch die Raumplanung widmen. Darüber hinaus ist die Lektüre dieser Veröffentlichung allen Lesern empfohlen, die an einem systematischen Überblick über das komplexe System der planerischen Steuerung des Einzelhandels in Deutschland interessiert sind.

\section{Literatur}

Blotevogel, H.H. (Hrsg.) (2002): Fortentwicklung des Zentrale-OrteKonzepts. Hannover. $=$ Forschungs- und Sitzungsberichte der ARL 217. 